\title{
Variation in spirometry utilization between trained general practitioners in practices equipped with a spirometer
}

\author{
PATRICK J. P. POELS ${ }^{1}$, TJARD R. J. SCHERMER ${ }^{1}$, ANNELIES JACOBS ${ }^{2}$, REINIER P. \\ AKKERMANS $^{1}$, JOLIET HARTMAN ${ }^{2}$, BEN A. M. BOTTEMA ${ }^{1,2}$ \& CHRIS VAN WEEL $^{1}$ \\ ${ }^{1}$ Department of General Practice, Radboud University Nijmegen Medical Centre, and ${ }^{2}$ Centre for Quality of Care Research, \\ Radboud University Nijmegen Medical Centre, The Netherlands
}

\begin{abstract}
Objective. To explore spirometry utilization among general practitioners and identify practitioner and practice-related factors associated with spirometry utilization. Design. Multivariate multilevel cross-sectional analysis of a questionnaire survey. Setting. Some 61 general practices involved in a spirometry evaluation programme in the Netherlands. All practices owned a spirometer and were trained to perform spirometry. Subjects. A total of 144 general practitioners and 179 practice assistants. Main outcome measures. Extent of spirometry utilization for five indications from national COPD/asthma guidelines, practitioner and practice-related factors associated with spirometry utilization. Results. The response rate was $97 \%$. General practitioners used spirometry mostly to evaluate treatment with inhaled steroids (58\%). Significant practitioner-related factors associated with spirometry utilization were: general practitioners' job satisfaction, general practitioners' general interest in research, and prior participation in spirometry training. Practice-related factors associated with spirometry utilization were: presence of a practice nurse, delegation of medical tasks to practice assistants, use of spirometry in different rooms, and use of protocols in practice. Conclusion. Practitioner- as well as practice-related factors were associated with the extent of spirometry utilization. In particular, it is essential to improve practice-related factors (e.g. presence of a practice nurse, more delegation of medical tasks to the practice assistant).
\end{abstract}

Key Words: Asthma, COPD, family practice, primary care, spirometry

In recent years the number of spirometers in primary care has increased. Currently general practitioners' (GPs) ownership of a spirometer varies between $60 \%$ and $80 \%$ in the UK $[1,2]$. In general practice, equipment is no longer a limiting factor for spirometry utilization as rather inexpensive and reliable electronic spirometers have become widely available. According to guidelines for general practice [3] and respiratory care [4], spirometry constitutes an essential tool to determine the presence and severity of airflow obstruction, and to distinguish between reversible and irreversible obstruction. The Dutch College of General Practitioners' guideline on COPD [5] states that availability of spirometry is an essential precondition for GPs to test and treat most patients with mild or moderately severe COPD.
Although spirometry is feasible in primary care, general practitioners (GPs) experience barriers that impede its utilization.

- Dutch GPs used spirometry mostly to evaluate a recently initiated treatment with inhaled steroids.

- Trained GPs with a special interest in research, with adequate resources and in a practice providing structured care, are more likely to use spirometry.

- In particular, practice-related factors (e.g. presence of a practice nurse, delegation of medical tasks) are primordial to improve spirometry. 
Carrying out spirometry in general practice seems justified in terms of test validity, provided that practice staff have been trained sufficiently [6]. This creates an essential precondition for implementation of spirometry in the general practice setting, but by no means guarantees actual integration of spirometry in the GP's management of respiratory diseases [7-9]. It seems that there are still barriers with regard to successful implementation of spirometry in primary care. Local factors like inadequate reimbursement of spirometry in own practice [10], and its general complexity to fit it into daily practice are well-documented common barriers that could explain a variation in spirometry utilization between GPs $[1,11]$. The variation in spirometry utilization seems also to be linked to practitioner-related factors (e.g. GPs' spirometry training level) and practicerelated factors (e.g. being in a group practice) [12]. Little is known about which of these factors are easily modifiable and essential to improve. The objective of the present study was to explore spirometry utilization among trained and wellequipped GPs. In order to give concrete direction to future reseach on this topic, we also identified practitioner- and practice-related factors that were associated with the extent of spirometry utilization by GPs.

Approval was provided by the medical ethics review board of Radboud University Nijmegen Medical Centre.

\section{Material and methods}

Design and data collection

A questionnaire survey was mailed to 61 practices involved in a spirometry evaluation programme [6]. In that study a pair of spirometric tests (laboratory and general practice) was performed twice in about seven study subjects per practice. The current questionnaire survey took place 14 months after GPs and practice assistants had been offered an initial spirometry training programme, to ensure that practices had enough time to implement spirometry for all patients in daily practice (not only for study purposes). All of these practices owned a spirometer (MicroLoop ${ }^{\circledR}$, Micro Medical Ltd, Rochester, Kent, UK), spirometry software (Spirare ${ }^{\circledR}$, Diagnostica Ltd, Oslo, Norway) and had at least one practice assistant employed who was trained to perform spirometry. (In Dutch primary care, practice assistants are professionally trained for administrative and clinical patient-directed support tasks).

\section{Questionnaires}

Discussion groups and interviews with experts in the fields were used to develop questionnaires to measure potential practitioner- and practice-related factors that may explain the extent of spirometry utilization by GPs. We developed separate questionnaires for GPs and practice assistants. First, we sent a questionnaire to a contact person (GP) in each practice to collect general information on the characteristics of the practice setting, practice organization and equipment, and information regarding the composition of the practice staff. Second, we sent to all GPs and practice assistants involved in these practices a questionnaire regarding the professional experience, general training level and continuous medical education, spirometry quality assurance, value of spirometry, and utilization of spirometry in daily practice (only for GPs). We used items in this questionnaire from a validated instrument [13]. Considerable effort was expended to achieve an optimal response. A €22 incentive was offered to practice staff for returning the questionnaires. We sent reminders to nonresponders at approximately four-week intervals, for a total of two mailings. Practices that did not respond to the reminders were telephoned by the researchers.

\section{Outcomes and analyses}

Spirometry utilization was assessed on the basis of GPs' self-reported utilization of spirometry for five indications for spirometry that are included in national GP guidelines for diagnosing and managing COPD and asthma (see Figure 1) $[3,5,14]$. For each indication GPs rated the extent to which they applied spirometry in their daily practice: $0=$ seldom or never; $1=$ sometimes; $2=$ often or always use of spirometry. A total sum score (range $0-10$ ) for these five indications was calculated.

The sum score was considered to reflect " $G P S$ ' spirometry utilization" and was used as the dependent variable in subsequent analyses. Because of the hierarchical structure of the study (GPs clustered within practices) we performed a multilevel analysis. In this analysis we accounted for the variability associated with each level of clustering. Analyses were performed in SAS V8.2 for Windows (SAS institute Inc, Cary USA 1999-2001) and were based on a mixed-effects model (PROC MIXED). In this model both fixed and random effects can be analysed. We used a random intercept model with practice as random variable and all other variables fixed. This means that we expected that the intercept varied randomly between practices and the other regression parameters in the model 


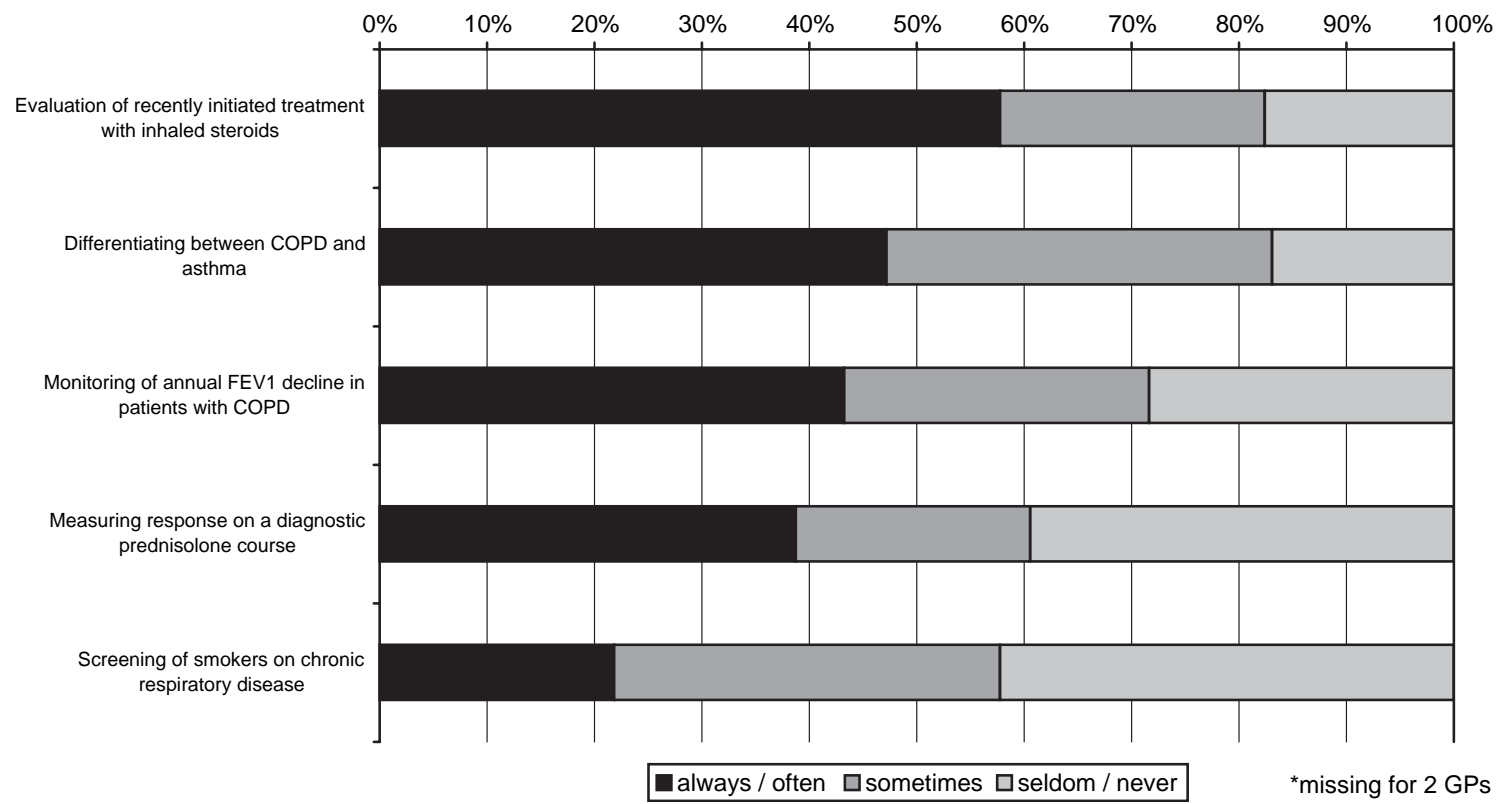

Figure 1. GPs' spirometry utilization for five indications that are included in the Dutch GP guidelines $\left(\mathrm{n}=144^{\star}\right)$.

had the same (fixed) value for each practice. The interpretation of the intercept and regression parameters is the same as in ordinary regression analyses, i.e. the value of each regression parameter (Beta) is corrected for the other variables in the model.

Univariate multilevel analyses were applied to assess the dependency of GPs' spirometry utilization on the explanatory variables. Multivariate multilevel analyses were applied with 23 explanatory variables. A backward elimination procedure was performed. Variables with a $\mathrm{p}$-value of $<0.05$ remained in the final model (see Table II). The interclass correlation coefficient (ICC) was assessed to give insight into the proportion of variance that was accounted for by practice level. Also, the fraction of explained variance at practice level and practitioner level was calculated.

\section{Results}

\section{Characteristics of general practices}

The response rate was $97 \%$ (59/61). Reasons for non-response of the practices remained unknown in one practice and one practice had merged recently with another practice that was not involved in the spirometry evaluation programme. In Table I we compare some characteristics of the general practices, GPs, and practice assistants involved in our study with national data from the Netherlands. Compared with the national figures, singlehanded practices were relatively underrepresented and group practices overrepresented among the practices in our study.

\section{Spirometry utilization}

GPs' spirometry utilization was normally distributed: mean 5.65 points (SD 2.47). Clustering of GPs within practices accounted for $16.8 \%$ of the total variation in GPs' spirometry utilization (ICC = 0.168). Figure 1 shows GPs' spirometry utilization for the five indications included in the Dutch national GP guidelines.

The indication for which the GPs reported the highest rate of spirometry utilization was "Evaluation of recently initiated treatment with inhaled steroids in COPD or asthma patients" (58\%). The indication with the lowest spirometry utilization rate was "Screening of smokers on chronic respiratory disease" $(22 \%)$.

\section{Practitioner- and practice-related factors and their association with spirometry utilization}

Table II shows the results of the stepwise multivariate multilevel analyses. The practitioner-related factors that were associated with GPs' spirometry utilization were GPs' job satisfaction $(\mathrm{p}=0.003)$, GPs' general interest in research $(p=0.01)$, and GPs' participation in the spirometry training during the study $(\mathrm{p}=0.02)$.

Practice-related factors associated with GPs'spirometry utilization were the presence of practice nurse support $(p<0.001)$, the extent of delegation of medical tasks to practice assistants $(p=0.003)$, use 


\section{P. F. P. Poels et al.}

Table I. Characteristics of the general practices, general practitioners, and practice assistants involved in the study (left) and from national data in the Netherlands (right): Values are means (SD) unless otherwise stated.

\begin{tabular}{|c|c|c|}
\hline General practices & $\mathrm{n}=59$ & $\mathrm{n}=4564^{1}$ \\
\hline \multicolumn{3}{|l|}{ Type of practice, $\%$} \\
\hline Single-handed & 33.9 & 60.7 \\
\hline Duo & 27.1 & 26.4 \\
\hline Group ( $\geq 3$ GPs) & 30.5 & 12.9 \\
\hline Multidisciplinary healthcare centre & 8.5 & - \\
\hline GPs, number per practice & $2.5(1.4)$ & NA \\
\hline Practice assistants, number per practice & $3.1(1.4)$ & NA \\
\hline Time since introduction of spirometry, years & $4.3(2.9)$ & NA \\
\hline General practitioners & $\mathrm{n}=144$ & $\mathrm{n}=8209^{1}$ \\
\hline Age, $\%<40$ years & 25.7 & 21 \\
\hline Professional experience, years & $14.3(8.2)$ & NA \\
\hline Gender, $\%$ female & 30.6 & 31.4 \\
\hline Patients per GP, number per practice & $1862(771)$ & 2392 \\
\hline Practice assistants & $\mathrm{n}=179$ & $\mathrm{n}=10000^{2}$ \\
\hline Age, $\%<40$ years & 61.5 & \pm 68 \\
\hline Professional experience, years & $10.7(7.4)$ & NA \\
\hline Gender, $\%$ female & 99.4 & 99 \\
\hline
\end{tabular}

${ }^{1}$ Data (1 January 2004) from the Netherlands Institute for Health Service Research (http://www.nivel.nl). ${ }^{2}$ Data (1 January 2004$)$ from the Dutch Association of Dokters Assistants (personal communication). NA = not available.

of spirometry in different rooms $(p=0.007)$ in the practice, task differentiation among GPs within the same practice $(p=0.01)$, and the use of protocols in practice $(p=0.01)$. The fraction of explained variance with this model was $26.3 \%$. Furthermore, $82.9 \%$ of all variance at practice level and $14.9 \%$ of all variance at GP level was explained.

\section{Discussion}

The results of this study indicate that GPs utilized spirometry mostly for diagnostic and monitoring purposes and seldom for screening purposes. We identified three practitioner- and five practice-related factors that were associated with the extent of spirometry utilization by GPs.

Table II. Results of stepwise multivariate multilevel analyses.

\begin{tabular}{|c|c|c|c|c|}
\hline Explanatory variable & Reference category & $\beta$ & $\mathrm{p}$ & $95 \% \mathrm{CI}$ \\
\hline \multicolumn{5}{|l|}{ Practitioner-related factors } \\
\hline Job satisfaction (subjective) & Point on sum score ${ }^{1}$ & 0.197 & 0.003 & $0.070-0.323$ \\
\hline General interest in scientific research & Non-participant & 0.997 & 0.01 & $0.238-1.759$ \\
\hline Spirometry training during the study [6] & Non-attender & 0.883 & 0.02 & $0.116-1.651$ \\
\hline \multicolumn{5}{|l|}{ Practice-related factors } \\
\hline Practice nurse support & $\mathrm{No}^{2}$ & 2.203 & $<0.001$ & $0.929-3.477$ \\
\hline Delegation medical tasks - practice assistants & $\%$ point delegated tasks & 0.042 & 0.003 & $0.015-0.069$ \\
\hline Spirometry used in different rooms & No & 1.116 & 0.007 & $0.313-1.918$ \\
\hline Task differentiation among GPs & No & -1.104 & 0.01 & $-1.956--0.252$ \\
\hline Use of protocols in practice & Point on sum score ${ }^{3}$ & 0.515 & 0.01 & $0.112-0.918$ \\
\hline
\end{tabular}

Explanatory variables are sorted by descending p-value. Explained fraction of variance; $\mathrm{R}^{2}=26.3 \%$. ${ }^{1}$ Sum score (range $0-10$ ) of five questions (Likert scale) concerning GP's satisfaction with available time for patients, work, continuous medical education, family, and leisure time. ${ }^{2}$ In Dutch primary care, practice nurses are professionally trained for support tasks, predominantly in chronic diseases (COPD $\&$ asthma or diabetes). They work under the supervision of a GP. They follow strict protocols for medical care and give education to patients. They do not order additional investigations. They are not allowed to refer patients. Nowadays, they are increasingly employed in multidisciplinary healthcare centres or group practices. ${ }^{3}$ Sum score (range $0-4$ ) of five questions (yes $=1$, no $=0$ ) with regard to the presence of protocols for visiting patients admitted to hospital; separate office hours for diabetes care or cardiovascular disease; invitation system for cervical cancer screening; invitation system for annual influenza vaccination. 


\section{Strengths and weaknesses of the study}

One of the strengths of our study was an excellent response rate of almost $100 \%$. Furthermore, in an opportunistic setting (participants in a study on spirometry) we analysed the effect of introduction of spirometry in daily practice on GPs' self-reported actual utilization. Through correction in the analyses for the fact that GPs were clustered in the same practices and may share one or more practice assistants, we could assess separately practitionerand practice-related factors that were associated with spirometry utilization. Practices were all equipped with a spirometer as an integral part of the evaluation. Consequently, the absence of a spirometer was not a limiting factor with regard to the implementation of spirometry. Generally, most trained GPs seem to prefer to perform spirometry in their own practice [15]. We took into consideration all these aspects in the setting of our study.

We could explain $26.3 \%$ of all variance in GPs' spirometry utilization, the dependent variable in our analysis. However, this subjective measure of goodness-of-fit also indicates that $73.7 \%$ of the variation could not be predicted with the current data. In particular the variance at GP level could not be explained by this model. Apparently, there are other (psychological) factors that influence utilization that have not been asked about in the questionnaires.

A weakness of the study is the external validity. We could only analyse GPs' perception of their actual use of spirometry once equipment was available and staff had been trained in its use. Due to selective participation of GPs with a general interest in research and the fact that - compared with national data - we included a relatively small proportion of single-handed practices our findings may not fully reflect the situation in Dutch general practice as a whole. Because no national data on spirometry ownership of general practices are available for the Netherlands, we do not know to what proportion of all practices our findings apply.

From a methodological point of view we accept that objective assessment of GPs' actual use of spirometry instead of the perception of use would have been more sophisticated. As there was an almost complete lack of studies in this area, we chose to explore spirometry utilization by GPs first by questionnaire. There have been contradictory reports as to the accuracy of physicians' self-reported adherence to guidelines in the literature. On the one hand, questionnaires tend to have moderate to high concordance with other - less subjective - measures of adherence [16]. On the other hand, clinicians' self-reported adherence rates may also exceed objective rates, which may result in an overestimation of adherence of up to $25 \%$ [17]. In our case, there is no reason to assume that the degree of overestimation of spirometry utilization - if indeed present would be different for the five separate indications for spirometry from the national guidelines for GPs that were studied. One could also wonder whether a consistent overestimation would have given different results with regard to the observed associations between practitioner- and practice-related factors and spirometry utilization rates. Although we used five indications for spirometry from guidelines to assess a total sum score, we do realize that the role of spirometry in diagnostics and monitoring of asthma is still controversial in daily practice with regard to best practice.

\section{Comparison with previous studies}

Generally, from this study and other studies $[1,18]$ spirometry seems to be underused for several indications in primary healthcare. The results of the current study indicate that GPs utilized spirometry in daily practice not only for diagnosis of respiratory diseases but also for management purposes. Specific utilization of spirometry for management purposes in primary care has been reported previously $[1,18]$. In line with these studies $[1,18]$ GPs' utilization of spirometry for screening purposes in asymptomatic smokers was very low $(22 \%)$, which seems legitimate considering the current view that widespread screening of smokers for the presence of airflow obstruction cannot be recommended at this time [19].

To the best of our knowledge this is the first study that assessed by means of multivariate multilevel analyses practitioner- and practice-related factors that were associated with spirometry utilization. Presently, only one study is available to mirror our results. O'Dowd et al. [12] determined physicianrelated and practice-related factors that were associated with owning a spirometer and use of spirometry in the evaluation of new asthma patients. Factors associated with frequent use of spirometry among GPs were ownership of a spirometer, GPs' belief that such testing provides data necessary for a diagnosis and, finally, a sufficient level of training to perform and interpret these tests. In our study all practices owned a spirometer but we also found an association between adequate training level to interpret tests $(\mathrm{p}=0.02)$ and actual utilization of spirometry by GPs.

\section{Possible implications for clinical practice}

The extent of spirometry utilization was associated with trained GPs with a special interest in research, 
with adequate resources (e.g. support staff and room space) and practices providing structured care to patients (e.g. use of protocols). To attain such an optimal situation in one's own practice we suggest having a special practice nurse for respiratory diseases employed in a practice. Special office hours for respiratory diseases attended by this practice nurse - under the supervision of a GP - will improve the service for these patients [20,21]. Second, the autonomy of practice assistants will increase by delegation of routine tasks from the GP to the practice assistant. Increased delegation of medical tasks was associated with more successful spirometry utilization. Third, the use of protocols in practice stimulates systematic working. Fourth, continuous spirometry education and training should be facilitated to maintain standards for GPs, practice assistants, and practice nurses [18]. Training of practice staff is preferably organized by non-commercial organizations (e.g. GPs' professional organizations).

\section{Conclusion and future research}

We conclude that trained GPs with a special interest in research, with adequate resources (support staff and room space) and in a practice providing structured care (protocols), were more likely to use spirometry in this study. If a GP lacks these conditions, it is essential to improve practice-related factors in particular (e.g. presence of a practice nurse, delegation of medical tasks to the practice assistant, and the use of protocols). This exploratory study adds to the current state of knowledge regarding the utilization of spirometry in general practice. The next step would be to verify our findings in a larger sample of all GPs in the Netherlands as well as in other countries, and preferably to measure the actual utilization of spirometry by GPs in patients with an indication for this particular lung function test.

\section{Acknowledgements}

The authors would like to thank their colleagues and nurses in the participating general practices, and Jonathan Honigh, MD, for data management. Boehringer Ingelheim BV is kindly acknowledged for allowing use of their spirometry training programmes for GPs and practice assistants.

This study was supported by grant 3.2.98.50 from the Netherlands Asthma Foundation.

\section{References}

[1] Jones RCM, Freegard S, Reeves M, Hanney K, Dobbs F. The role of the practice nurse in the management of chronic obstructive pulmonary disease (COPD). Prim Care Respir J 2001;10:106-8.

[2] Bolton CE, Ionescu AA, Edwards PH, Faulkner TA, Edwards SM, Shale DJ. Attaining a correct diagnosis of COPD in general practice. Respir Med 2005;99:493500.

[3] Geijer RMM, Thiadens HA, Smeele IJM, Sachs APE, Bottema BJAM, van Hensbergen W, et al. Dutch College of General Practitioners Guideline on COPD and adult asthma: Diagnosis. Huisarts Wet 2001;44:107-17.

[4] Pauwels RA, Buist AS, Calverley PM, Jenkins CR, Hurd SS Global strategy for the diagnosis, management, and prevention of chronic obstructive pulmonary disease. NHLBI/ WHO Global Initiative for Chronic Obstructive Lung Disease (GOLD) Workshop summary. Am J Respir Crit Care Med 2001;163:1256-76.

[5] Geijer RMM, van Schayck CP, van Weel C, Sachs AP, Bottema BJAM, Smeele IJM, et al. Dutch College of General Practitioners Guideline on COPD: treatment. Huisarts Wet 2001;44:207-19.

[6] Schermer TR, Jacobs JE, Chavannes NH, Hartman J, Folgering HT, Bottema BJ, et al. Validity of spirometric testing in a general practice population of patients with chronic obstructive pulmonary disease (COPD). Thorax 2003;58:861-6.

[7] Kesten S, Chapman KR. Physician perceptions and management of COPD. Chest 1993;104:254-8.

[8] Choudhury A, Griffiths CJ, Packe GE. Diagnosis of chronic obstructive pulmonary disease (COPD) - are guidelines being implemented in primary care. Eur Respir J 2001; 18(Suppl 33):347s.

[9] Decramer M, Bartsch P, Pauwels R, Yernault JC. Management of COPD according to guidelines: A national survey among Belgian physicians. Monaldi Arch Chest Dis 2003; 59:62-80.

[10] Chan B, Anderson G, Dales RE. Spirometry utilization in Ontario: Practice patterns and policy implications. Can Med Assoc J 1997;156:169-76.

[11] White P. Spirometry and peak expiratory flow in the primary care management of COPD. Prim Care Respir J 2004;13: $5-8$.

[12] O'Dowd LC, Fife D, Tenhave T, Panettieri RA Jr. Attitudes of physicians toward objective measures of airway function in asthma. Am J Med 2003;114:391-6.

[13] Van den Hombergh P, Grol R, van den Hoogen HJ, van den Bosch WJ. Assessment of management in general practice: validation of a practice visit method. Br J Gen Pract 1998;48: 1743-50.

[14] Geijer RMM, van Hensbergen W, Bottema BJAM, van Schayck CP, Sachs APE, Smeele IJM, et al. Dutch College of General Practitioners Guideline on adult asthma: Treatment. Huisarts Wet 2001;44:153-64.

[15] Dowson LJ, Yeung A, Allen MB. General practice spirometry in North Staffordshire. Monaldi Arch Chest Dis 1999; $54: 186-8$.

[16] Garber MC, Nau DP, Erickson SR, Aikens JE, Lawrence JB. The concordance of self-report with other measures of medication adherence: A summary of the literature. Med Care 2004;42:649-52.

[17] Adams AS, Soumerai SB, Lomas J, Ross-Degnan D. Evidence of self-report bias in assessing adherence to guidelines. Int J Qual Health Care 1999;11:187-92. 
[18] Eaton T, Withy S, Garrett JE, Mercer J, Whitlock RM, Rea HH. Spirometry in primary care practice: The importance of quality assurance and the impact of spirometry workshops. Chest 1999;116:416-23.

[19] Smith-Sivertsen T, Rortveit G. Should general practitioners screen smokers for COPD? Scand J Prim Health Care 2004; 22:196-201.

[20] Griffiths C, Feder G, Wedzicha J, Foster G, Livingstone A, Singh Marlowe G. Feasibility of spirometry and reversibility testing for the identification of patients with chronic obstructive pulmonary disease on asthma registers in general practice. Respir Med 1999;93:903-8.

[21] Van Son I, Vrijthoef H, Crebolder H, van Hoef L, Beusmans G. Support for the general practitioner: An exploration of the effect of the practice nurse on the care of asthma, COPD and diabetes patients. Huisarts Wet 2004;47: $15-21$. 